\title{
ON THE EXISTENCE OF SOLUTIONS OF NONLINEAR EQUATIONS
}

\author{
MICHAL FEČKAN \\ (Communicated by Jeffrey B. Rauch)
}

\begin{abstract}
Results on the existence of solutions are derived for asymptotically quasilinear, nonlinear operator equations. Applications are given to implicit nonlinear integral equations.
\end{abstract}

\section{INTRODUCTION}

The purpose of this paper is to study the existence of a solution for the operator equation

$$
L(x)=N(x)+h,
$$

where $L: H \rightarrow H$ is continuous, $N: H \rightarrow H$ is continuous compact, $h \in H$ and $H$ is a real Hilbert space with the inner product $(\cdot, \cdot)$ and norm $|\cdot|$. We assume that $L$ is asymptotically linear at infinity and $N$ is asymptotically quasilinear at infinity. The asymptotic quasilinearity of $N$ at infinity roughly speaking means the approximability of $N$ as $x \rightarrow \infty$ by a family $\mathcal{M}$ of bounded linear maps. We are motivated by the papers [6], [9] for introducing this notion. Under some conditions on $\mathcal{M}$ we associate to $\mathcal{M}$ a generalized Leray-Schauder degree in the sense of the papers [2], [10]. Then we show that (1.1) is solvable for any $h \in H$. This is a generalization of the well-known results about asymptotically linear operators (see $[4],[7],[8],[15])$.

We also study the case when the operators $L$ and $N$ leave a wedge in $H$ invariant. We find sufficient conditions for the existence of a solution of (1.1) in that wedge. This is an extension of similar results of the papers [1], [5], [9], [11].

Applications are given to implicit nonlinear integral equations. We are motivated by the papers [12]-[14] to study implicit integral equations, because boundary value problems of differential equations are studied in [14] when highest-order derivatives are not solvable as well as there are certain stimulations from physics in [12], [13]. Finally, we note that results of this paper can be also applied to problems studied in $[2],[10]$.

Received by the editors July 8, 1994 and, in revised form, November 9, 1994

1991 Mathematics Subject Classification. Primary 45M20, 47H05, 47H17.

Key words and phrases. Pseudomonotone mappings, integral equations, nonnegative solutions. 


\section{Solvability of (1.1)}

The following definitions will be needed in the sequel (see [2, p. 946]).

A mapping $f: H \rightarrow H$ is:

- monotone (denote $f \in M O N)$, if $(f(u)-f(v), u-v) \geq 0$ for all $u, v \in H$;

- pseudomonotone $(f \in P M)$, if for any sequence $\left\{u_{n}\right\}$ in $H$ with $u_{n} \rightarrow u$ (weak convergence) and $\overline{\lim }\left(f\left(u_{n}\right), u_{n}-u\right) \leq 0$, it follows that $f\left(u_{n}\right) \rightarrow f(u)$ and $\left(f\left(u_{n}\right), u_{n}\right) \rightarrow(f(u), u)$;

- of class $S_{+}\left(f \in S_{+}\right)$, if for any sequence $\left\{u_{n}\right\}$ in $H$ with $u_{n} \rightarrow u$ and $\varlimsup\left(f\left(u_{n}\right), u_{n}-u\right) \leq 0$, it follows that $u_{n} \rightarrow u$;

- compact $(f \in C O M P)$, if it is continuous and for any bounded sequence $\left\{u_{n}\right\}$ in $H$ the sequence $\left\{f\left(u_{n}\right)\right\}$ has a convergent subsequence;

- completely continuous $(f \in C C)$, if for any sequence $\left\{u_{n}\right\}$ in $H$ with $u_{n} \rightarrow u$, it follows that $f\left(u_{n}\right) \rightarrow f(u)$;

- bounded, if it takes any bounded set of $H$ into a bounded set.

We note that the following relations hold between the above definitions

$$
\begin{aligned}
& C C \subset C O M P, \quad S_{+} \subset P M, \quad M O N \subset P M ; \\
& f_{1} \in S_{+}, f_{2} \in C O M P \Rightarrow f_{1}-f_{2} \in S_{+} ; \\
& f_{1} \in P M, f_{2} \in C C \Rightarrow f_{1}-f_{2} \in P M ; \\
& f \in P M \Rightarrow f+\varepsilon \mathbb{I} \in S_{+} \forall \varepsilon>0,
\end{aligned}
$$

where $\mathbb{I}: H \rightarrow H$ is the identity map .

In what follows, we shall assume that the mappings are bounded and continuous.

Definition 2.1. A map $N: H \rightarrow H$ is said to be asymptotically quasilinear at infinity if there is a mapping $N_{\infty}: H \rightarrow 2^{H}$ such that

i) $N_{\infty}(u)$ is a closed nonnempty subset of $H$ for any $u \in H$;

ii) $\alpha N_{\infty}(u)=N_{\infty}(\alpha u) \forall \alpha \geq 0, \forall u \in H$;

iii) $\overline{\bigcup_{u \in S} N_{\infty}(u)}$ is a compact subset for $S=\{u \in H|\quad| u \mid=1\}$;

iv) $N_{\infty}$ is upper-semicontinuous;

v) $\forall \varepsilon>0 \exists R>0$ such that $\forall u,|u| \geq R, \exists w_{u} \in N_{\infty}(u)$ satisfying

$$
\frac{\left|N(u)-w_{u}\right|}{|u|} \leq \varepsilon
$$

$N_{\infty}$ is said to be the asymptote of $N$.

Remark 2.2. Let us take

$$
N_{\infty}(u)=\{K u\} \quad \forall u \in H
$$

for a compact linear map $K: H \rightarrow H$. Then the assumptions i)-iv) of Definition 2.1 are satisfied trivially. The assumption $\mathrm{v}$ ) expresses the usual asymptotic linearity at infinity. So a mapping $L: H \rightarrow H$ is said to be asymptotically linear at infinity if there is a bounded linear map (the so-called asymptote of $L$ ) $L_{\infty}: H \rightarrow H$ such that

$$
\lim _{|u| \rightarrow \infty} \frac{\left|L(u)-L_{\infty} u\right|}{|u|}=0 .
$$


Theorem 2.3. Suppose $L, N$ are asymptotically linear, quasilinear at infinity with the asymptotes $L_{\infty} \in S_{+}, N_{\infty}$, respectively. Assume $L-N \in P M$ and there is a compact linear map $A: H \rightarrow H$ such that

$$
0 \notin L_{\infty} u-\lambda N_{\infty}(u)-(1-\lambda) A u \quad \forall u \in S, \forall \lambda \in[0,1] .
$$

Then (1.1) has a solution for any $h \in H$.

Proof. For simplicity, we consider the case $h=0$. First, we show the existence of $\delta>0, R_{0}>0$ such that

$$
\begin{aligned}
& \left|\lambda L(u)-\lambda N(u)+(1-\lambda)\left(L_{\infty} u-A u\right)\right| \geq \delta \\
& \forall u,|u| \geq R_{0}, \quad \forall \lambda \in[0,1] .
\end{aligned}
$$

For this purpose, we compute

$$
\begin{aligned}
& \lambda L(u)-\lambda N(u)-(1-\lambda) A u+(1-\lambda) L_{\infty} u \\
& =|u|\left(L_{\infty} \frac{u}{|u|}-\lambda \frac{w_{u}}{|u|}-(1-\lambda) A \frac{u}{|u|}+\lambda \frac{L(u)-L_{\infty} u}{|u|}+\lambda \frac{w_{u}-N(u)}{|u|}\right),
\end{aligned}
$$

where $w_{u}$ is from Definition 2.1. Now we show that there is $c_{1}>0$ such that

$$
\begin{aligned}
& \left.\mid L_{\infty} z-\lambda w_{z}-(1-\lambda) A z\right) \mid \geq c_{1} \\
& \forall z \in S, \forall w_{z} \in N_{\infty}(z), \quad \forall \lambda \in[0,1] .
\end{aligned}
$$

If it is not true, then there is a sequence $\lambda_{i} \in[0,1], z_{i} \in S, w_{z_{i}} \in N_{\infty}\left(z_{i}\right)$ such that $\lambda_{i} \rightarrow \lambda_{0}$ and

$$
L_{\infty} z_{i}-\lambda_{i} w_{z_{i}}-\left(1-\lambda_{i}\right) A z_{i} \rightarrow 0 .
$$

We can assume $z_{i} \rightarrow z_{0}$ (the weak convergence) and $w_{z_{i}} \rightarrow \bar{w}$ (see the assumption iii) of Definition 2.1). So we have both $A z_{i} \rightarrow A z_{0}$, since $A$ is compact linear, and

$$
L_{\infty} z_{i}-\lambda_{0} \bar{w}-\left(1-\lambda_{0}\right) A z_{0} \rightarrow 0
$$

Since $L_{\infty} \in S_{+}$, we can assume $z_{i} \rightarrow z_{0}$. This gives

$$
L_{\infty} z_{0}-\lambda_{0} \bar{w}-\left(1-\lambda_{0}\right) A z_{0}=0
$$

Finally, we know $w_{z_{i}} \rightarrow \bar{w}, w_{z_{i}} \in N_{\infty}\left(z_{i}\right)$ and $z_{i} \rightarrow z_{0}$. The assumptions i) and iv) of Definition 2.1 imply $\bar{w} \in N_{\infty}\left(z_{0}\right)$. Since (2.5) contradicts (2.1), (2.4) is proved.

We return to (2.3). By the assumption ii) of Definition 2.1 we have

$$
\frac{w_{u}}{|u|} \in N_{\infty}\left(\frac{u}{|u|}\right)
$$

in (2.3). We see now that (2.4) gives (2.2) for $R_{0}$ sufficiently large. 
According to the assumptions $L-N \in P M, L_{\infty} \in S_{+}, A \in C O M P$ and (2.0), we have that

$$
\lambda(L-N)+(1-\lambda)\left(L_{\infty}-A\right)+\varepsilon \mathbb{I} \in S_{+} \quad \forall \lambda \in[0,1],
$$

where $\mathbb{I}: H \rightarrow H$ is the identity map and $\varepsilon>0$. So the following relations hold:

$$
\operatorname{deg}\left(L-N+\varepsilon \mathbb{I}, B_{R_{0}}, 0\right)=\operatorname{deg}\left(L_{\infty}-A, B_{R_{0}}, 0\right) \neq 0,
$$

where $B_{R_{0}}=\left\{u \in H|\quad| u \mid<R_{0}\right\}, \varepsilon>0$ is sufficiently small and deg is the generalized Leray-Schauder degree in the sense of the papers [2], [10]. This gives the solvability of $L(u)+\varepsilon u=N(u)$ in $B_{R_{0}}$ for any $\varepsilon>0$ sufficiently small. By passing to the limit $\varepsilon \rightarrow 0_{+}$and using $L-N \in P M$, we obtain the solvability of $L(u)=N(u)$. The proof is finished.

Remark 2.4. If $N_{\infty}(\cdot)$ are, moreover, convex and $A u \in N_{\infty}(u) \forall u \in H$, then (2.1) is simplified to

$$
0 \notin L_{\infty} u-N_{\infty}(u) \quad \forall u \in S .
$$

This case happens in the following way. Let $\mathcal{M}$ be a nonempty compact convex subset of the set of all compact linear maps from $H$ into $H$. We put

$$
N_{\infty}(u)=\{K u \mid K \in \mathcal{M}\} \quad \forall u \in H .
$$

It is clear that the assumptions i)-iv) of Definition 2.1 hold as well as the convexity of $N_{\infty}(\cdot)$. Now we can take $A=K_{0}$ for any fixed $K_{0} \in \mathcal{M}$. So we can associate the degree $\operatorname{deg}\left(L_{\infty}-K_{0}, B_{1}, 0\right)$ to $\mathcal{M}$. Here $B_{1}=\{u \in H|\quad| u \mid<1\}$. We note that $\operatorname{deg}\left(L_{\infty}-K_{0}, B_{1}, 0\right) \neq 0$ is constant in $K_{0} \in \mathcal{M}$. If the remaining assumptions of Theorem 2.3 hold, then (1.1) is solvable for any $h \in H$.

Finally, if $N$ is asymptotically linear at infinity, i.e. $\mathcal{M}=\{B\}$ for a compact linear map $B$, then (2.1) is simplified to

$$
0 \neq L_{\infty} u-B u \quad \forall u \neq 0 .
$$

Then Theorem 2.3 is an extension of [7, Theorem XI.1] and the proof of this theorem is related to a proof of that one.

Now we look for special solutions of (1.1). Let $C \subset H$ be a wedge, i.e. $C$ is a closed, nonempty, convex subset of $H$ such that $\lambda C \subset C \forall \lambda \geq 0$. We know (see [3, p. 71]) that there is a continuous retraction $\eta: H \rightarrow C$, the so-called metric projection, such that $\eta(\lambda x)=\lambda \eta(x) \forall \lambda \geq 0, \forall x \in H$ and $|\eta(x)| \leq|x| \forall x \in H$.

We are interested in the existence of solutions of (1.1) in $C$. So in the rest of this section, we solve the equation

$$
L(x)=N(x), \quad x \in C,
$$

where $N \in C O M P$ satisfies $N(C) \subset C$ and $L \in M O N$ is such that $(L+\varepsilon \mathbb{I})(C)=C$ for any $\varepsilon>0$ sufficiently small. Here $\mathbb{I}$ is the identity map.

We suppose

(H1) $L$ is asymptotically linear at infinity with the asymptote $L_{\infty} \in S_{+}$satisfying $L_{\infty} x=0 \Rightarrow x=0$ and $L_{\infty}(C)=C$.

(H2) $N$ is asymptotically quasilinear at infinity with the asymptote $N_{\infty}$ satisfying $N_{\infty}(u) \subset C \forall u \in C$. 
Theorem 2.5. Assume that (H1), (H2) hold and $L-N \in P M$. If for any $x \in$ $C, 0<\lambda \leq 1$, the condition $0 \in L_{\infty} x-\lambda N_{\infty}(x)$ implies $x=0$, then (2.6) has a solution.

Proof. We solve

$$
L(x)+\varepsilon x=N(\eta(x))
$$

for $\varepsilon>0$ small. We know partly that $L+\varepsilon I$ is strongly monotone (i.e. $\left(\left(L\left(x_{1}\right)+\right.\right.$ $\left.\left.\varepsilon x_{1}\right)-\left(L\left(x_{2}\right)+\varepsilon x_{2}\right), x_{1}-x_{2}\right) \geq \varepsilon\left|x_{1}-x_{2}\right| \forall x_{1}, x_{2} \in H$ ), so it is invertible (see [3, p. 100]) and partly that $(L+\varepsilon \mathbb{I})(C)=C$. Hence any solution of $(2.7)$ belongs to C.

Now we verify the assumption (2.1) with $A=0$ for the map $L+\varepsilon \mathbb{I}-N(\eta)$ with $\varepsilon>0$ sufficiently small, i.e. we intend to apply Theorem 2.3 to (2.7). For this purpose, we claim that the asymptote of $N(\eta)$ is $N_{\infty}(\eta)$. Indeed, the boundedness of $N$ and the assumptions of Definition 2.1 imply that for any $\omega>0$ there is a constant $c(\omega)>0$ such that for any $u \in H$ there is $w_{u} \in N_{\infty}(u)$ satisfying

$$
\left|N(u)-w_{u}\right| \leq \omega|u|+c(\omega) .
$$

Since for any bounded $B \subset H$, the compactness of $\overline{N_{\infty}(B)}$ follows from the assumptions i)-iv) of Definition 2.1, we can clearly take

$$
c(\omega)=\sup \left\{|N(u)|+|w| \quad|u \in H ;| u \mid \leq R ; w \in N_{\infty}(u)\right\},
$$

where $R$ is established by the assumption v) of Definition 2.1 when $\omega$ is considered instead of $\varepsilon$.

Hence we have

$$
\left|N(\eta(u))-w_{\eta(u)}\right| \leq \omega|\eta(u)|+c(\omega) \leq \omega|u|+c(\omega) .
$$

Lastly, the validity of the assumptions i)-iv) of Definition 2.1 for $N_{\infty}(\eta(\cdot))$ can be easily verified. The claim is proved.

If (2.1) is not true for this case with $A=0$, then there is a sequence $\varepsilon_{i}>0, u_{i} \in$ $S, \lambda_{i} \in[0,1]$ such that $\varepsilon_{i} \rightarrow 0, u_{i} \rightarrow u_{0}, \lambda_{i} \rightarrow \lambda_{0}$ and

$$
0 \in L_{\infty} u_{i}+\varepsilon_{i} u_{i}-\lambda_{i} N_{\infty}\left(\eta\left(u_{i}\right)\right) .
$$

So there is $w_{i} \in N_{\infty}\left(\eta\left(u_{i}\right)\right)$ such that

$$
0=L_{\infty} u_{i}+\varepsilon_{i} u_{i}-\lambda_{i} w_{i} .
$$

This equality gives

$$
L_{\infty} u_{i}-\lambda_{0} w_{i} \rightarrow 0
$$

By the assumption iii) of Definition 2.1, we can assume $w_{i} \rightarrow w$. Hence

$$
L_{\infty} u_{i}-\lambda_{0} w \rightarrow 0 .
$$

By the condition $L_{\infty} \in S_{+}$we can assume $u_{i} \rightarrow u_{0}$. Since $w_{i} \in N_{\infty}\left(\eta\left(u_{i}\right)\right)$, we obtain both $w \in N_{\infty}\left(\eta\left(u_{0}\right)\right)$ (see the assumptions i) and iv) of Definition 2.1) and 
$L_{\infty} u_{0}=\lambda_{0} w$. The assumptions $(H 1-2)$ imply the invertibility of $L_{\infty}$ and $w \in C$. Hence we obtain $u_{0} \in C$. Finally, we see that the equation (2.8) gives

$$
0 \in L_{\infty} u_{0}-\lambda_{0} N_{\infty}\left(u_{0}\right)
$$

for some $u_{0} \in S \cap C$. This contradicts our assumptions. So (2.1) holds for (2.7). Hence Theorem 2.3 is applicable to (2.7) for any $\varepsilon>0$ sufficiently small. The proof of Theorem 2.3 gives a constant $M>0$ such that (2.7) has a solution $x_{\varepsilon},\left|x_{\varepsilon}\right| \leq M$. We already know $x_{\varepsilon} \in C$. By using both the pseudomonotony of $L-N$ and the weak closeness of $C$, we obtain the desired solution. The proof is finished.

Remark 2.6. If $L \in M O N$ holds also in Theorem 2.3, then $L-N+\varepsilon \mathbb{I} \in S_{+}$for any $\varepsilon>0$ (see (2.0)). Now, let the assumption $L-N \in P M$ be dropped in Theorem 2.5 and let this assumption be replaced by $L \in M O N$ in Theorem 2.3. Then the assumptions of both Theorems 2.3 and 2.5 , modified in this way, imply only the almost solvability of $L(u)=N(u)$ in the sense that

$$
0 \in \overline{(L-N)(H)}, \quad \text { respectively } \quad 0 \in \overline{(L-N)(C)} .
$$

Indeed, by the ends of the proofs of Theorems 2.3 and 2.5, there is a constant $M>0$ such that both equations $L(u)+\varepsilon u=N(u)$ and $L(u)+\varepsilon u=N(u), u \in C$, have solutions $x_{\varepsilon},\left|x_{\varepsilon}\right| \leq M$, for any $\varepsilon>0$ sufficiently small. So the claim is proved.

Remark 2.7. The assumption $L-N \in P M$ in Theorems 2.3 and 2.5 is satisfied provided that $L \in P M$ and $N \in C C$.

\section{IMPLICIT INTEGRAL EQUATIONS}

In this section, we illustrate the above abstract results for certain integral equations of the type

$$
p(x, u(x))=\int_{0}^{1} G(x, t) m(t, u(t)) d t+f(x), \quad x \in[0,1],
$$

where $p, m \in C([0,1] \times \mathbb{R}, \mathbb{R}), G \in L_{2}([0,1] \times[0,1], \mathbb{R})$ and $f \in C([0,1], \mathbb{R})$. Moreover, we suppose that $p$ is nondecreasing in $u \in \mathbb{R}$. We are motivated by the papers [12]-[14] to study implicit integral equations.

We assume that there are constants $\alpha>0, \beta_{2} \geq \beta_{1}$ satisfying

$$
\lim _{|u| \rightarrow \infty}|p(x, u)-\alpha u| /|u|=0 \quad \text { uniformly in } x \in[0,1] ;
$$

$$
\beta_{1} \leq \liminf _{|u| \rightarrow \infty} m(x, u) / u \leq \limsup _{|u| \rightarrow \infty} m(x, u) / u \leq \beta_{2} \quad \text { uniformly in } x \in[0,1] .
$$

Theorem 3.1. Assume that $(3.2-3)$ hold and, moreover, suppose that $p$ is increasing in $u$, i.e.

$$
\left(p\left(x, u_{1}\right)-p\left(x, u_{2}\right)\right)\left(u_{1}-u_{2}\right)>0 \quad \forall x \in[0,1], u_{1} \neq u_{2} .
$$


If there is a constant $\delta>0$ such that the equation

$$
\alpha u(x)=\int_{0}^{1} G(x, t) \phi(t) u(t) d t
$$

has no nonzero solution for any $\phi \in L_{2}([0,1], \mathbb{R})$ satisfying $\beta_{1}-\delta \leq \phi(\cdot) \leq \beta_{2}+\delta$, then (3.1) has a solution.

Proof. We apply Theorem 2.3 and Remark 2.4 by putting

$$
\begin{aligned}
& H=L_{2}([0,1], \mathbb{R}), \quad L(u)=p(\cdot, u), \\
& N(u)=\int_{0}^{1} G(\cdot, t) m(t, u(t)) d t, \quad L_{\infty} u=\alpha u \\
& \mathcal{M}=\left\{u \rightarrow \int_{0}^{1} G(\cdot, t) \phi(t) u(t) d t \quad \mid \phi \in L_{2}([0,1], \mathbb{R}) ; \beta_{1}-\delta \leq \phi(\cdot) \leq \beta_{2}+\delta\right\}, \\
& N_{\infty}(u)=\{K u \mid K \in \mathcal{M}\} .
\end{aligned}
$$

It is clear that $L \in M O N, N \in C O M P$. We also know that the strict monotony of $p$ in $u$ implies $L \in S_{+}$(see [2], [10]). So $L-N \in P M$ (see (2.0)).

Now we verify the assumptions of Definition 2.1. The assumption i) follows partly from the weak compactness of the set

$$
\left\{\phi u \mid \phi \in L_{2}([0,1], \mathbb{R}) ; \beta_{1}-\delta \leq \phi(\cdot) \leq \beta_{2}+\delta\right\}
$$

in $L_{2}([0,1], \mathbb{R})$ for any $u \in L_{2}([0,1], \mathbb{R})$ fixed, and partly from the compactness of the map $u \rightarrow \int_{0}^{1} G(\cdot, t) u(t) d t$. So $N_{\infty}(\cdot)$ are compact subsets. The assumption ii) is clear. Since the set

$$
\left\{\left.\phi u\left|(\phi, u) \in L_{2}([0,1], \mathbb{R}) \times L_{2}([0,1], \mathbb{R}) ;\right| u\right|_{L_{2}}=1, \beta_{1}-\delta \leq \phi(\cdot) \leq \beta_{2}+\delta\right\}
$$

is bounded in $L_{2}([0,1], \mathbb{R})$ and the map $u \rightarrow \int_{0}^{1} G(\cdot, t) u(t) d t$ is compact, the assumption iii) is proved. The assumption iv) is clear, since $N_{\infty}(\cdot)$ are compact subsets and $\mathcal{M}$ is a set of uniformly bounded linear mappings. To prove v), we have by (3.3) that $m$ can be decomposed as

$$
m(x, u)=m_{1}(x, u) u+m_{2}(x, u),
$$

where $m_{1}, m_{2}$ are continuous, $m_{2}(\cdot, \cdot)$ is bounded on $[0,1] \times \mathbb{R}$ and $\beta_{1}-\delta \leq m_{1}(\cdot, \cdot) \leq$ $\beta_{2}+\delta$. Now it is clear that we can take

$$
w_{u}=\int_{0}^{1} G(x, t) m_{1}(t, u(t)) u(t) d t \quad \forall u \in L_{2}([0,1], \mathbb{R})
$$


in the assumption v) of Definition 2.1. So $N_{\infty}$ is the asymptote of $N$. Similarly, by using (3.2) we can show that $L_{\infty}$ is the asymptote of $L$.

It remains to verify

$$
0 \notin L_{\infty} u-N_{\infty}(u) \quad \forall u \in S
$$

for this case. If it is not true, then there is $u \in L_{2}([0,1], \mathbb{R})$ such that

$$
\alpha u(x)=\int_{0}^{1} G(x, t) \phi(t) u(t) d t
$$

for some $\phi \in L_{2}([0,1], \mathbb{R})$ satisfying $\beta_{1}-\delta \leq \phi(\cdot) \leq \beta_{2}+\delta$. But the assumption of this theorem implies $u=0$. So (2.1) is valid. We see that all assumptions of Theorem 2.3 are satisfied. The proof is finished.

Theorem 3.2. Assume that asymptotic behaviors $(3.2-3)$ hold only for $u \rightarrow+\infty$. Suppose in addition that

$$
\begin{gathered}
p(\cdot, 0)=0, \quad f(\cdot) \geq 0, \quad G(\cdot, \cdot) \geq 0, \quad m(\cdot, u) \geq 0 \forall u \in[0,+\infty) ; \\
p \text { is increasing in } u .
\end{gathered}
$$

If there is a constant $\delta>0$ such that the equation

$$
\alpha u(x)=\int_{0}^{1} G(x, t) \phi(t) u(t) d t
$$

has no nonzero nonnegative solution for any $\phi \in L_{2}([0,1], \mathbb{R})$ satisfying $0 \leq \phi(\cdot) \leq$ $\beta_{2}+\delta$, then (3.1) has a nonnegative solution.

Proof. We follow the above proof. Since we are interested in nonnegative solutions of (3.1), we can assume, by modifying $p, m$ for $u \rightarrow-\infty$, the validity of (3.2-3) with $\beta_{1}=0$. We take

$$
C=\left\{u \in L_{2}([0,1], \mathbb{R}) \quad \mid \quad u \geq 0 \text { almost everywhere on }[0,1]\right\} .
$$

It is clear that $N(C) \subset C,(L+\varepsilon \mathbb{I})(C)=C$. Finally, the remaining assumptions of Theorem 2.5 are verified in the same way as above. The difference is only that we now take

$$
\mathcal{M}=\left\{u \rightarrow \int_{0}^{1} G(\cdot, t) \phi(t) u(t) d t \quad \mid \phi \in L_{2}([0,1], \mathbb{R}) ; 0 \leq \phi(\cdot) \leq \beta_{2}+\delta\right\} .
$$

The proof is finished.

By applying Remark 2.6 we obtain the following

Theorem 3.3. If the strict monotony of $p$ in $u$ is dropped in Theorems $3.1-2$, i.e. $p$ is only nondecreasing in u, then (3.1) is almost solvable in these theorems.

We complete this paper by presenting two examples. 
Example 1. Consider the equation

$$
(p(x, u))^{\prime}=m(x, u), \quad u(0)=a,
$$

where $p, m$ possess the properties in (3.1).

Theorem 3.4. If $(3.2-3)$ hold and $p$ is increasing in $u$, then (E1) has a solution, i.e. there is $u \in C([0,1], \mathbb{R})$ such that $p(\cdot, u) \in C^{1}([0,1], \mathbb{R})$ and $u$ satisfies $(\mathrm{E} 1)$.

Proof. We apply Theorem 3.1, since (E1) has the form

$$
p(x, u)=\int_{0}^{x} m(t, u(t)) d t+p(0, a) .
$$

The linear equation in Theorem 3.1, for this case, has the form

$$
\alpha u(x)=\int_{0}^{x} \phi(t) u(t) d t,
$$

where $\phi \in L_{2}([0,1], \mathbb{R})$ satisfies $\beta_{1}-\delta \leq \phi(\cdot) \leq \beta_{2}+\delta$. By using the Gronwall lemma, this equation has only the zero solution. The proof is finished.

Example 2. Consider the equation

$$
(p(x, u))^{\prime \prime}=m(x, u), \quad u(0)=u(1)=0,
$$

where $p, m$ possess the properties in (3.1).

Theorem 3.5. Assume that $(3.2-3)$ hold with

$$
\beta_{1}>-\pi^{2}(n+1)^{2} \alpha, \quad \beta_{2}<-\pi^{2} n^{2} \alpha
$$

for a nonnegative integer number $n$. If $p$ is increasing in $u$, then (E2) has a solution; i.e. there is $u \in C([0,1], \mathbb{R})$ such that $p(\cdot, u) \in C^{2}([0,1], \mathbb{R})$ and $u$ satisfies $(\mathrm{E} 2)$.

Proof. By observing

$$
(p(x, u))^{\prime \prime}=(p(x, u)-p(0,0)-x(p(1,0)-p(0,0)))^{\prime \prime},
$$

we consider that $p(0,0)=p(1,0)=0$. We apply Theorem 3.1, since (E2) has the form

$$
p(x, u)=\int_{0}^{1} G(x, t) m(t, u(t)) d t,
$$

where $G$ is the Green function of $w^{\prime \prime}=z, w(0)=w(1)=0$. The linear equation in Theorem 3.1, for this case, has the form

$$
\alpha u(x)=\int_{0}^{1} G(x, t) \phi(t) u(t) d t,
$$

where $\phi \in L_{2}([0,1], \mathbb{R})$ satisfies $\beta_{1}-\delta \leq \phi(\cdot) \leq \beta_{2}+\delta$. This equation is equivalent to

$$
\alpha u^{\prime \prime}=\phi u, \quad u(0)=u(1)=0 .
$$

Since $\beta_{1}>-\pi^{2}(n+1)^{2} \alpha$ and $\beta_{2}<-\pi^{2} n^{2} \alpha$, it is well-known (see [3]) that the equation (3.8) has only the zero solution for a sufficiently small constant $\delta>0$. The proof is finished. 


\section{REFERENCES}

1. H. Amann, Fixed points of asymptotically linear maps in ordered Banach spaces, J. Functional Analysis 14 (1973), 162-171. MR 50:3019

2. J. Berkovits \& V. Mustonen, An extension of Leray-Schauder degree and applications to nonlinear wave equations, Diff. Int. Equations 3 (1990), 945-963. MR 91j:35179

3. K. Deimling, "Nonlinear Functional Analysis", Springer-Verlag, Berlin, 1985. MR 86j:47001

4. M. Fečkan, Critical points of asymptotically quadratic functions, Annal. Polon. Math LXI.1 (1995), 63-76.

5. M. Fečkan, Nonnegative solutions of nonlinear integral equations, Comment. Math. Univ. Carolinae (to appear).

6. M. Fečkan, An inverse function theorem for continuous mappings, J. Math. Anal. Appl. 185 (1994), 118-128. MR 95b:58017

7. R.E. Gaines \& J. Mawhin, "Coincidence Degree, and Nonlinear Differential Equations", Lec. Not. Math. 568, Springer-Verlag, Berlin, 1977. MR 58:30551

8. A. Granas, On a certain class of nonlinear mappings in Banach spaces, Bull. Acad. Polon. Sci. Ser. Sci. Math. Astronom. Phys. 9 (1957), 867-871. MR 19:968e

9. R. Guzzardi, Positive solutions of operator equations in the non-differentiable case, in "Contemporary Mathematics", Vol. 21, 1983, 137-146. MR 85e:47088

10. A. Kittilä, On the topological degree for a class of mappings of monotone type and applications to strongly nonlinear elliptic problems, Ann. Acad. Sci. Fenn. Ser. A I Math. Dissertationes 91 (1994). MR 95e: 47079

11. M.A. Krasnoselskii, "Positive Solutions of Operator Equations", Noordhoff, Groningen, 1964. MR 31:6107

12. W. Okrasiński, On a non-linear convolution equation occurring in the theory of water percolation, Annal. Polon. Math. 37 (1980), 223-229. MR 82b:76056

13. W. Okrasiński, On the existence and uniqueness of nonnegative solutions of certain nonlinear convolution equation, Annal. Polon. Math. 36 (1979), 61-72. MR 80f:45010

14. W.V. Petryshyn, Solvability of various boundary value problems for the equation $x^{\prime \prime}=$ $f\left(t, x, x^{\prime}, x^{\prime \prime}\right)-y$, Pacific J. Math. 122 (1986), 169-195. MR 87g:34022

15. J. Santanilla, Existence of nonnegative solutions of a semilinear equation at resonance with linear growth, Proc. Amer. Math. Soc. 105 (1989), 963-971. MR 89j:34054

Department of Mathematical Analysis, Faculty of Mathematics and Physics, ComeniUs University, Mlynská dolina, 84215 BratislaVa, Slovakia

E-mail address: Michal.Feckan@fmph.uniba.sk 